Liczner Grzegorz, Wiciński Michal, Cadelski Karol, Malinowski Bartosz. Is the determination of bone turnover markers and vitamin D useful in the diagnosis of degeneration spine disease? Journal of Education, Health and Sport. 2021;11(9):259-271. eISSN 2391-8306. DOI http://dx.doi.org/10.12775/JEHS.2021.11.09.032

https://apcz.umk.pl/JEHS/article/view/JEHS.2021.11.09.032

https://zenodo.org/record/5515131

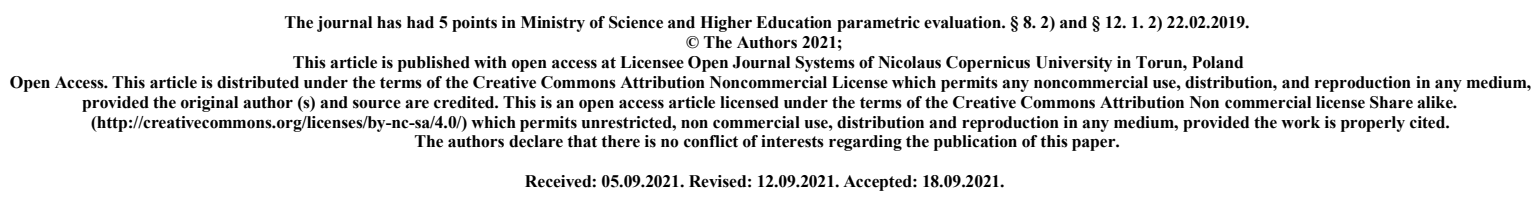

\title{
Is the determination of bone turnover markers and vitamin D useful in the diagnosis of degeneration spine disease?
}

\author{
Grzegorz Liczner, Michał Wiciński, Karol Cadelski, Bartosz Malinowski
}

\author{
Department of Pharmacology and Therapeutics, Faculty of Medicine, Collegium Medicum in Bydgoszcz, \\ Nicolaus Copernicus University, M. Curie 9, 85-090 Bydgoszcz, Poland
}

\begin{abstract}
Degenerative disease of the lumbar spine is one of the most common diseases of the human musculoskeletal system and a significant public health problem. The main manifestation of degenerative spine disease is back pain, which in most cases is treated symptomatically rather than causally. The diagnosis of degenerative spine disease has been previously based on medical history and diagnostic imaging. Recently, much attention has been devoted to the biochemical indicators of bone and cartilage metabolism, which are well understood among patients with osteoporosis. Bone turnover markers indicate the metabolic activity of bone and cartilage. They occur in blood serum, urine, and synovial fluid in the form of collagen-derived proteins or enzymes, involved in the formation or resorption of bone and cartilage tissue. The study below was aimed at assessing the usefulness of determining the markers of bone turnover and cartilage damage in the blood serum of people with degenerative disease of the lumbar spine diagnosed by MRI. The biochemical diagnostics included the measurement of inflammatory parameters. The results show that the degenerative disease of the spine is local, accompanied by inflammation, and vitamin D deficiency is among the factors leading to its development.
\end{abstract}

Key words: degenerative spine disease, back pain, markers of bone turnover, markers of cartilage damage, vitamin D.

\section{Epidemiology of the degenerative spine disease}

The high incidence of degenerative spine disease is a significant social and medical problem in terms of the patient and public health. The World Health Organization lists osteoarthritis among the top 10 health problems.[1] In the light of the latest research, back pain is considered the leading ailment of the skeletal system. The worldwide prevalence is $12-30 \%$. Estimated data show that $75-85 \%$ of people suffer or will suffer from back pain at least once in their lifetime. There is now an increasing tendency for the first symptoms of back pain to appear earlier in life. The etiology of back pain is multifactorial and depends, among other factors on, age, weight, lifestyle, smoking, physical activity, and diet.[2,3] Pathomorphological studies by Schmorl and Junghanns from 1936, Coventry from 1945, Hirsch from 1960, and Tiedjen and Müller from 2001 showed that almost all people over the age of 30 have noticeable degenerative changes in the spine, manifested mainly in the intervertebral disc. Signs of advanced degenerative disease of the spine, presenting as spondylosis or myelopathy, are visible in imaging studies in most people with back pain over 65 and affect almost $100 \%$ of people between 80 and 90 years old, which means that the frequency of this disease is closely age-related.[4] 


\section{Pathophysiology of the degenerative spine disease}

Degenerative spine disease has many stages, it affects not only the intervertebral disc but many structures adjacent to it. Pathologies in the muscular system may destabilize the spine, predispose to the development of a degenerative disease or aggravate its symptoms.[5] Many malignant neoplasms metastasize to the axial skeleton, especially the vertebral bodies. This process results in deformities or compression fractures, leading to static disorders of the spine perceived as severe pain. It is not uncommon for a vertebral compression fracture to be the first symptom of malignant disease. [6,7] There is a close correlation between the development of degenerative changes in the spine and the age of the patients. Due to the vertical body posture adopted by humans and the associated excessive pressure, a poorly nourished intervertebral disc is exposed to premature wear. It is associated with a gradual deterioration in the metabolism of the intervertebral disc, mainly as a result of its dehydration.[8] According to Keyes and Compère, water accounts for $88 \%$ of the intervertebral discs of newborns, and only $70 \%$ of those over the age of 70.[9] Water is an integral structural component of the disc and at the same time a carrier of nutrients and metabolic products. Therefore, its deficiency impairs the metabolism of the disc, strengthens its biochemical and anatomical changes. These changes are manifested by the loss of mucopolysaccharides, proteoglycans, and collagen proteins, resulting in the fibrosis of gel-like nucleus pulposus and a decrease in the elasticity of the fibrous ring. When the degenerated disc is unable to perform its role properly, its functions are compensated by adjacent structures, becoming overloaded, rebuilt, or damaged, which significantly disturbs the mechanics of the spine and leads to the development of a degenerative disease.[10,11] The first stage of degenerative spine disease is repeated overload of the intervertebral disc (discopathy). It consists of a gradual loss of water (dehydration) and the formation of raptures within the fibrous ring, which results in a lowering of the disc height and often a hernia of the nucleus pulposus. Simultaneously with the development of discopathy, occurs gradual damage to the other motor structures of the spine, i.e. the vertebral bodies, intervertebral joints, yellow and longitudinal ligaments. Changes involving these structures are known as spondylosis.[12,13] The degenerative process of the yellow ligaments is manifested by their hypertrophy and thickening, while in the longitudinal ligaments by a decrease in elasticity and an increase in their stretchability.[14] As a consequence, degenerative spondylolisthesis may develop, which together with vertebral body osteophytes, proliferative lesions of the articular processes, and progressive intervertebral disc bulging contribute to the narrowing of the spinal canal. The degenerative processes are most often located in the cervical and lumbosacral sections of the spine.[15]

\section{Brief characteristics of bone turnover markers and vitamin D}

Bone turnover markers are indicators of the metabolic activity of bone and cartilage. They take the form of either non-enzymatic proteins produced during bone formation or resorption or enzymes that regulate both. Their concentration can be measured in the blood serum, urine, and synovial fluid of people suffering from metabolic bone diseases, which include, among others, osteoarthritis, including degenerative spine disease. The markers of bone formation include bone fraction of alkaline phosphatase (BALP), osteocalcin (OC), procollagen type $1 \mathrm{~N}$-terminal propeptide (P1NP), procollagen type $1 \mathrm{C}$-terminal propeptide (P1CP). Bone resorption markers include: hydroxyproline (HYP), pyridnoline (PYD) and deoxypyridinoline (DPD), crosslinked N-telopeptide of type 1 collagen (NTX1), cross-linked C-telopeptide of type 1 collagen (CTX1), carboxy-terminal telopeptide of type 1 collagen (1CTP) and tartrate-resistant acid phosphatase 5b (TRAP-5b). The cross-linked C-telopeptide of type 2 collagen (CTX2) and the cartilage oligomeric matrix protein (COMP) are indicators of cartilage damage. Four hormones are among the regulators of calcium and phosphate metabolism in the human body: parathyroid hormone, calcitonin, vitamin $\mathrm{D}$, and cortisol.

Currently, procollagen type $1 \mathrm{~N}$-terminal propeptide (P1NP) is the most widely described marker of bone formation in the literature. It is formed during the process of extracellular maturation of collagen, which consists of cutting N-terminal amino acid residues from type 1 procollagen, synthesized by osteoblasts and fibroblasts of soft tissues and skin. The severed products are initially in trimeric forms which eventually are degraded to monomeric form in the blood serum. Trimeric P1NP is removed by hepatic uptake while the monomeric form is eliminated by the kidneys. P1NP shows low daily and individual variability and is stable at room temperature. The available tests measure monomeric and trimeric forms (total P1NP) or only trimeric forms (intact P1NP).[16] The monomeric form of P1NP is dependent on glomerular filtration and accumulates 
in chronic kidney disease.[17] High levels of P1NP are observed physiologically during the intensive growth of children and adolescents and in diseases with high bone metabolism, such as Paget's disease, hyperparathyroidism, and osteoblastic bone metastases.[18] According to the Working Group of the International Osteoporosis Foundation, P1NP is a recognized predictor of fractures in osteoporosis, and its serum concentration reflects the sensitivity to pharmacotherapy.[19]

Vitamin D comprises a group of fat-soluble steroidal compounds of exo- or endogenous origin, the common feature of which is a four-ring structure, enriched with a side chain. Vitamin D precursors in the human body come from self-synthesis in the keratinocytes of the spinous and basal layer of the epidermis from 7-dehydrocholesterol, which is converted into cholecalciferol under the influence of UVB rays. Vitamin D can also be supplied with the plant (ergocalciferol) or animal (cholecalciferol) food. Regardless of their origin, each of these forms is hydroxylated at position 25 in the liver and position $1 \alpha$ in the kidney to the active form of vitamin $\mathrm{D}-1,25(\mathrm{OH})_{2} \mathrm{D}$ (calcitriol). The synthesis of biologically active vitamin $\mathrm{D}$ depends on many factors and is strictly regulated. Its production is stimulated by parathyroid hormone and inhibited by calcium, phosphates, fibroblast growth factor-23 (FGF-23), and a high concentration of active vitamin $\left(1,25(\mathrm{OH})_{2} \mathrm{D}\right)$ ).[20] The effect of vitamin D on target cells is related to the intracellular VDR receptor (Vitamin D Receptor), present not only in osteoblasts but also in the cells of the renal tubules, intestinal epithelium, or in the blood vessel wall. The ubiquity of the VDR receptor determines the pleiotropic effect of vitamin D. VDR, forming a heterodimer with the retinoic acid receptor (RXR), regulates the transcription of at least 229 genes, the expression of which is associated with cell growth and differentiation, and in diseases including cancer, diabetes, and arthritis.[21] In the small intestine, vitamin D induces the transcription of calcium transporting genes (TRPV6, Calbindin-D9k, and PMCA1b), resulting in increased calcium absorption. The physiological role of vitamin $\mathrm{D}$ is to regulate the absorption and transport of essential minerals, especially calcium, phosphorus, and magnesium, which are important for maintaining proper bone structure. Vitamin D deficiency is one of the causes of rickets in children, whereas in adults it leads to osteomalacia or premature degenerative changes in the joints. Several studies focus now on determining the correlation between serum vitamin D levels and bone mineral density and the initiation and progression of osteoarthritis.[22,23]

Parathyroid hormone is an 84 amino acid polypeptide produced by the parathyroid glands and counteracts calcitonin. It is responsible for increased calcium reabsorption in the kidney and a simultaneous reduction of tubular reabsorption of phosphates. Its role in the kidneys is also revealed by supporting the maturation of vitamin $25(\mathrm{OH}) \mathrm{D}$ to $\left(1,25(\mathrm{OH})_{2} \mathrm{D}\right)$. [24] The impact of PTH on calcium and phosphate metabolism depends mainly on the kinetics of the hormone concentrations in the blood serum. The anabolic effect of PTH includes a direct effect on osteoblasts and an indirect effect related to the activation of growth factors (IGF-1) and the inhibition of growth factor antagonists such as sclerostin. Parathyroid hormone initiates bone formation through 4 pathways: enhancement of osteoblast proliferation and differentiation, inhibition of osteoblast apoptosis, reduction of the negative effects of the peroxisome proliferator activator receptor (PPAR) on osteoblast differentiation, and enhancement of the Wnt/ $\beta$-catenin pathway, which appears to be crucial for osteogenesis.[25] Hyperparathyroidism, characterized by an overproduction of PTH inadequate to the systemic needs, strongly inhibits collagen formation, leading to increased osteolysis and the release of calcium into the blood. Bone symptoms are caused by generalized micro-architectural deterioration of bone tissue or by focal defects such as osteitis fibrosa cystica. This leads to pain and deformities of the spine, and sometimes pathological fractures of the ribs, vertebrae, and long bones.[26] Pulsatile administration of parathyroid hormone in low doses, by contrast, stimulates collagen synthesis, which has been used in the treatment of osteoporosis. Teriparatide is a recombinant 1-34 N-terminal fragment of the parathyroid hormone molecule obtained by biotechnology using E. coli bacteria, which is administered subcutaneously to patients with severe osteoporosis. It reduces the risk of vertebral and other bone fractures in men and women, including steroidinduced osteoporosis.[27] The efficacy of teriparatide in the treatment of osteoporosis was evaluated using one of the markers of bone formation. Clinical trials have shown an increase in P1NP (procollagen type $1 \mathrm{~N}$ terminal propeptide) in patients with osteoporosis within 3 months of starting treatment compared to those receiving placebo. Moreover, the concentration of P1NP positively correlates with an increase in the activity of the skeleton, assessed in radioisotope studies and bone histomorphometry. Patients treated with teriparatide typically experienced an increase in P1NP $>10 \mu \mathrm{g} / \mathrm{L}$ from baseline, while those treated with placebo did not present such changes.[28] The regulation of calcium and phosphate metabolism is presented in the figure below. 


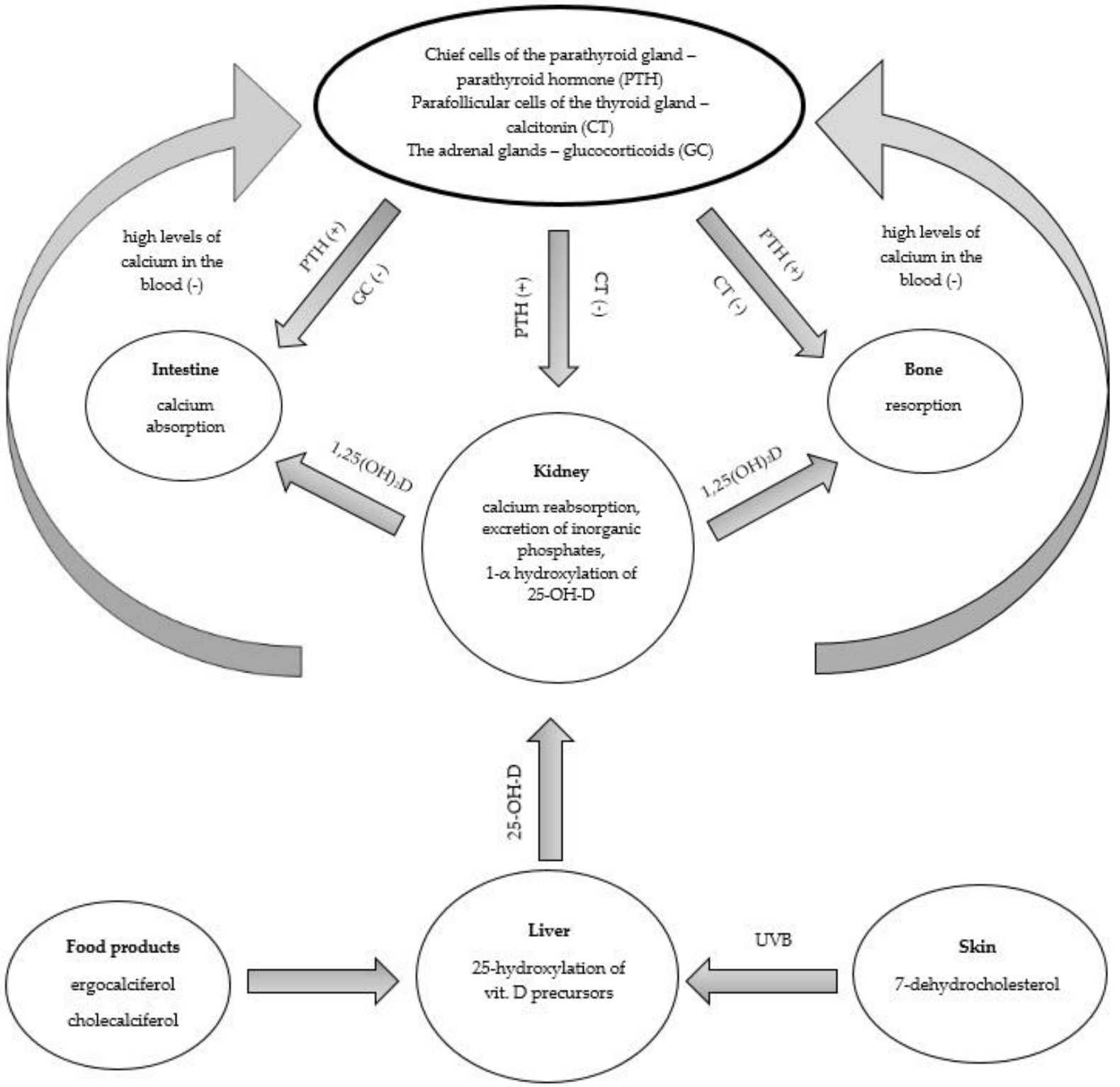

Figure 1. Regulation of calcium homeostasis.

In degenerative joint diseases, apart from noticeable changes in the skeletal system, progressive damage to the cartilage tissue is also observed. One of the initial symptoms of osteoarthritis is the degeneration of the extracellular matrix associated with the loss of the biomechanical function of cartilage. The extracellular matrix constitutes $90 \%$ of the cartilage mass and consists mainly of water, glycosaminoglycans (hyaluronic acid, chondroitin sulfate, heparan sulfate), proteins (collagen, fibronectin), and lipids.[29] Type II collagen is the main protein of cartilage. It forms a rich network of fibrils in it, giving the cartilage the appropriate shape, flexibility, and resistance to stretching.[30] In addition to collagen, the cartilage matrix consists of a high molecular weight proteoglycan and cartilage oligomeric matrix protein (COMP). Cartilage oligomeric matrix protein is a large pentameric glycoprotein that interacts with many extracellular matrix proteins in cartilage and other tissues. It participates in the secretion of collagen, chondrocyte proliferation and the mechanical strength of cartilage, ligaments and tendons. Mutations associated with COMP relate to disorders of the storage of this protein in the endoplasmic reticulum of chondrocytes. Retention of COMP in the reticulum increases oxidative and inflammatory processes, leading to chondrocyte death and loss of bone growth ability.[31] It is considered that the breakdown products of the cartilage extracellular matrix may serve as markers of the rate of destruction of cartilage tissue. These include the cross-linked C-telopeptide of type 2 collagen (CTX2), resulting from the breakdown of type 2 collagen chains that build cartilage, and the COMP protein. The CTX2 protein, since it is 
excreted by the kidneys, is most often determined in urine, while the COMP protein is obtained from the blood serum.[32] Garnero et al. were among the first to report that in patients with osteoarthritis of the knees, an increased concentration of CTX2 in the urine is also accompanied by an increased concentration of the COMP protein in the blood serum.[33] In the following years, attempts were made to search for markers of cartilage damage that could serve as a sensitive indicator of the severity of degenerative changes in joints, sometimes preceding bone changes. In 2015-2018 at The First Affiliated Hospital, Guangzhou Medical University, a study was conducted on 90 patients diagnosed with osteoarthritis, whose concentration of CTX2 and YKL-40 in the blood serum was assessed before and after the use of glucosamine sulfate and diacerein. YKL-40 belongs to the family of glycoside hydrolases that occur, among others, in chondrocytes. Measurements were made using immunoenzymatic techniques. It was noted that serum concentrations of CTX2 and YKL-40 tended to decline during treatment. The combined determination of these markers may therefore improve the sensitivity of the early diagnosis of osteoarthritis.[34]

Aim of the study: To assess the activity of the above-described markers of bone turnover (P1NP, COMP, CTX2) and vitamin D in the blood serum of people affected by degenerative disease of the lumbar spine.

\section{Material and methods}

Biochemical tests of blood serum were performed based on the consent of the Bioethics Committee at Ludwik Rydygier Collegium Medicum in Bydgoszcz, UMK in Toruń No. 353/2019 of May 14, 2019. 93 adults were included in the study, 30 of which belonged to the control group and 63 people to the study group. The control group consisted of healthy people who had not reported back pain so far and had not been diagnosed with any chronic disease, including degenerative spine disease. The study group consisted of people complaining of back pain of varying intensity and duration with a degenerative spine disease found simultaneously in imaging tests (MRI of the lumbar spine). Apart from the underlying disease, they did not report any other chronic diseases. Both in the control group and the study group, liver and kidney diseases were disqualifying factors, as the metabolism of most of the measured proteins depends on the efficiency of these organs, which could significantly affect the obtained results. Using the ELISA method at the Department of Pharmacology and Therapy of Ludwik Rydygier Collegium Medicum in Bydgoszcz, UMK in Torun, the concentration of the following proteins was determined: P1NP, COMP, CTX2, PTH and vitamin D. The characteristic of the groups included in the study are presented in the table below. 


\section{Results}

Table No. 1. Characteristic of the group of people included in the study

\begin{tabular}{|c|c|c|c|c|c|}
\hline group & & $\begin{array}{r}\text { height } \\
\text { (cm) }\end{array}$ & $\begin{array}{c}\text { body weight } \\
\text { (kg) }\end{array}$ & $\begin{array}{c}\text { BMI } \\
\left(\mathrm{kg} / \mathrm{m}^{2}\right)\end{array}$ & $\begin{array}{c}\text { age } \\
\text { (years) }\end{array}$ \\
\hline \multirow[t]{4}{*}{ study } & average & 169,86 & 78,97 & 27,2997 & 55,13 \\
\hline & $\mathrm{SD}$ & 9,064 & 16,218 & 4,91599 & 13,259 \\
\hline & median & 170,00 & 79,00 & 27,3400 & 56,00 \\
\hline & $\mathrm{n}$ & 63 & 63 & 63 & 63 \\
\hline \multirow[t]{4}{*}{ control } & average & 168,73 & 80,33 & 28,1887 & 58,70 \\
\hline & $\mathrm{SD}$ & 8,395 & 14,281 & 4,45917 & 15,663 \\
\hline & median & 167,50 & 80,50 & 28,0650 & 63,50 \\
\hline & $\mathrm{N}$ & 30 & 30 & 30 & 30 \\
\hline
\end{tabular}

The differences between the groups (study vs. control) in terms of age, BMI, weight and height are not statistically significant and do not differ much from the overall mean. 


\begin{tabular}{|c|c|c|c|c|c|c|c|}
\hline & \multicolumn{3}{|c|}{ study group } & \multicolumn{3}{|c|}{ control group } & \multirow{2}{*}{$\mathbf{p}$} \\
\hline & average & median & SD & average & median & SD & \\
\hline CTX2 pg/mL & 763,74 & 720,06 & 326,97 & 756,24 & 689,13 & 391,08 & 0,966 \\
\hline P1NP ng/mL & 455,88 & 268,95 & 425,18 & 563,63 & 443,34 & 450,52 & 0,226 \\
\hline COMP ng/mL & 36,83 & 37,32 & 12,34 & 30,61 & 30,42 & 15,06 & 0,054 \\
\hline Vitamin D ng/mL & 28,646 & 26,105 & 10,706 & 23,410 & 19,054 & 12,128 & 0,016 \\
\hline Calcium mmoL/L & 2,5202 & 2,5300 & 0,10318 & 2,3643 & 2,3600 & 0,08784 & $<0,001$ \\
\hline PTH pg/mL & 4,29 & 0,00 & 11,27 & 9,24 & 0,00 & 17,42 & 0,299 \\
\hline CRP mg/L & 3,42 & 1,40 & 6,805 & 1,17 & 0,62 & 1,775 & $<0,001$ \\
\hline
\end{tabular}

The increased concentration of P1NP, CTX2 and COMP in the blood serum of people from both groups proves that the development of the degenerative disease of the spine is associated with increased metabolism of bone and cartilage tissue. Statistically significant differences between the groups (study vs. control) were found in the concentration of vitamin D, calcium and CRP protein. Higher levels of total calcium and vitamin D were observed in the study group. This is likely due to vitamin D supplementation by people with back pain. It promotes increased absorption of calcium from the gastrointestinal tract, which increases its concentration in blood serum. Hypercalcemia associated with degenerative spine disease may also result from excessive release of calcium from the bones due to hormone stimulation (PTH) and intensive remodeling of bone tissue. Both the control group and the study group had normal serum parathyroid hormone levels. Vitamin D deficiency appears to be one of the causes of degenerative spine disease. Moreover, people diagnosed with degenerative spine disease have a higher concentration of CRP than healthy people. The box plots on the following pages compare vitamin D, calcium, and CRP concentrations between the groups. 

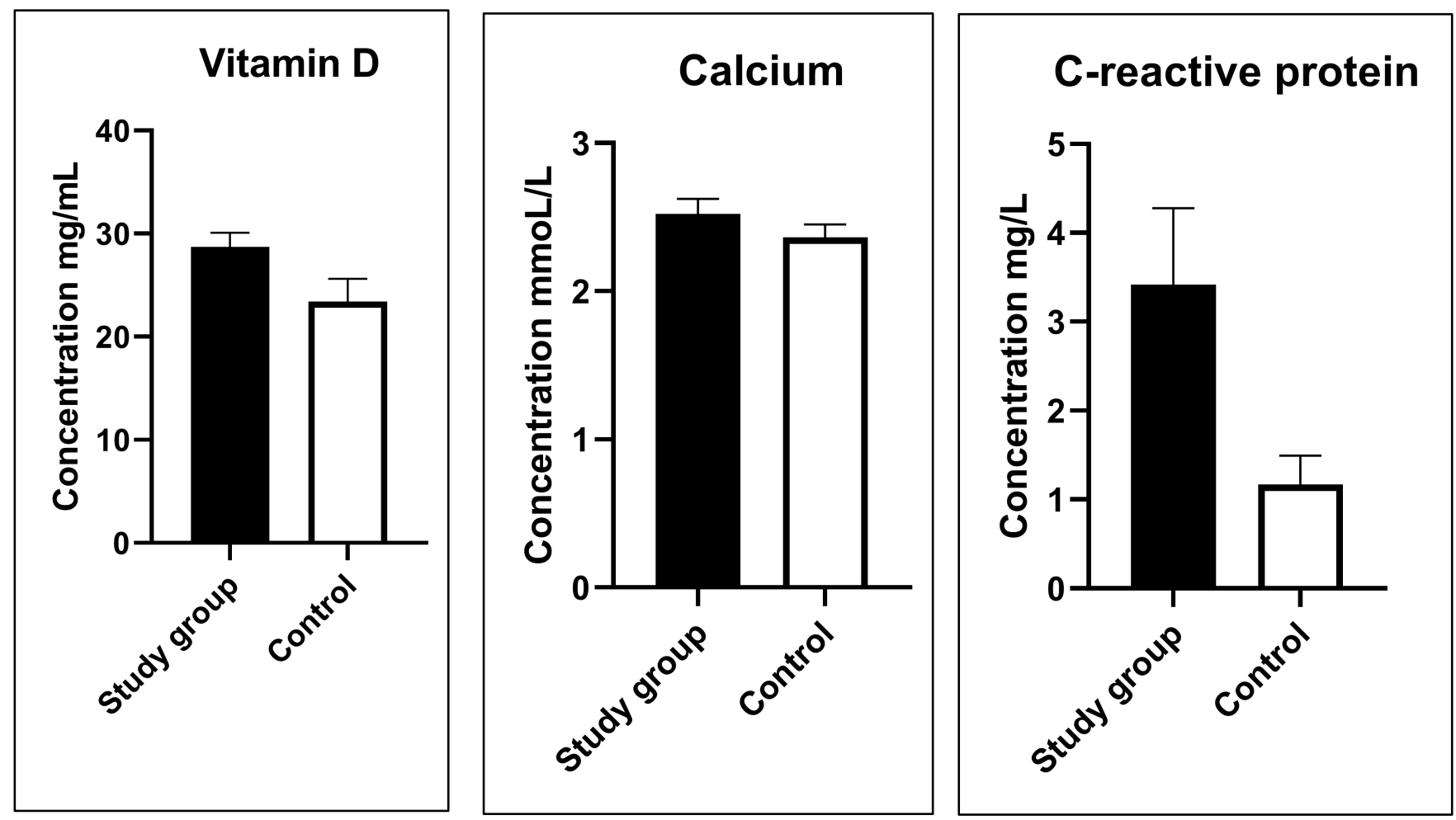

Figure 2. Comparison of groups for vitamin D, calcium and CRP protein

\section{Discussion}

Most of the reports on bone turnover markers refer to locally advanced degenerative joint diseases involving one or two adjacent joints, in which their concentration was tested mainly in the synovial fluid, alternatively in the urine. Degenerative spine disease seems to be a systemic multi-joint disease. Our team, therefore, attempted to determine the concentration of bone turnover markers in the blood serum of people suffering from this disease. We determined using the ELISA method: P1NP, CTX2, COMP and PTH. In addition, we determined the concentration of CRP protein. There are many reports on the usefulness of determining selected indicators of bone and cartilage remodeling in degenerative joint diseases. Research on their potential use in clinical practice continues to this day. Young-Min et al. presented the possibility of comparing the concentration of CTX2 in urine and cartilage oligomeric matrix protein (COMP) in blood serum or synovial fluid with the advancement of degenerative changes in the joints.[35,36] The conducted studies showed a correlation between the concentration of COMP in the blood serum and the severity of joint pain in people over 45 years of age without previously diagnosed degenerative changes in imaging tests.[37] The concentration of COMP also correlated with the advancement of degenerative changes in the knee joints in classic X-ray examinations.[38] In the tests performed by our team, we determined the concentration of CTX2 and COMP in the blood serum of healthy people and those suffering from degenerative spine disease. However, no statistically significant differences have been found between the two groups in terms of these proteins. Perhaps it results from a greater degree of remodeling of bone than cartilage tissue in degenerative spine disease comparing to other degenerative joints diseases. According to Garnero et al. and Reijman et al., patients with a high concentration of CTX2 in the urine are characterized by a much faster progression of degenerative changes in the hip joints.[39,40,41] Garnero also reports high levels of CTX2 in urine in patients with rheumatoid arthritis.[42] Moreover, Jung et al. found that urinary concentration of CTX2 is higher in people with coxarthrosis than in people with gonarthrosis, and in both of these diseases, the concentration of this marker significantly exceeds the values reported in healthy people.[43] CTX2 is measured increasingly in the synovial fluid, especially when the degenerative disease is locally advanced. According to Lohmander et al., the concentration of CTX2 in the synovial fluid of people suffering from any type of osteoarthritis is much 
higher than in healthy people and decreases during treatment, both in the synovial fluid and urine.[44] In the research conducted by our team, we found two statistically significant differences between the groups (study vs control) - in the concentration of vitamin $\mathrm{D}(28,646 \mathrm{ng} / \mathrm{mL}$ vs $23,410 \mathrm{ng} / \mathrm{mL})$ and calcium $(2,52 \mathrm{mmol} / \mathrm{L}$ vs $2,36 \mathrm{mmol} / \mathrm{L}$ ). They were higher in the case of people from the study group. This may result from more frequent supplementation of vitamin D preparations by people with diagnosed degenerative spine disease than by healthy people. People suffering from metabolic diseases of the skeletal system most often take a synthetic precursor of the active metabolite of vitamin D3 (alfacalcidol) and ready calcium preparations. The consequence of taking vitamin $\mathrm{D}$ is then an increase in the level of calcium in the blood serum of these people. Other causes of hypercalcemia were excluded by determining PTH - the main hormone that increases the concentration of calcium in the blood serum. Vitamin D deficiency may therefore be one of the causes of degenerative spine disease. The lack of an adequate supply of vitamin D limits the absorption and metabolism of calcium, which is necessary for the proper mineralization of bone tissue. The elderly and obese people are the most vulnerable to vitamin D deficiency. The main cause of vitamin D deficiency in humans is the lack of exposure to sunlight. Few food products contain vitamin D naturally, and foods fortified with vitamin D are often insufficient to meet the body's current needs. Vitamin D deficiency causes rickets in children, leads to osteopenia and osteoporosis in adults, and is associated with an increased risk of certain types of cancers, autoimmune diseases, high blood pressure and infectious diseases. The optimal serum level of 25hydroxyvitamin $\mathrm{D}$ is $75-125 \mathrm{nmol} / \mathrm{L}$ or $30-50 \mathrm{ng} / \mathrm{mL}$.[45] According to the meta-analysis conducted by Pereira-Santos et al., the prevalence of vitamin D deficiency was 35\% higher in obese subjects compared to the eutrophic group and $24 \%$ higher than in the overweight group, which is consistent with the results of our study.[46] Glover et al. in their study found that obese people with normal or high vitamin D values show better tolerance to the load than those with vitamin D deficiency, which was confirmed in the lower limb functional load test.[47] Therefore, they should develop osteoarthritis later than people with vitamin D deficiency. Zhang et al., in turn, noticed that progression of osteoarthritis of the knee joint depends, among others, on vitamin D deficiency.[48] We analyzed the concentrations of bone turnover markers and cartilage tissue damage in the blood serum between groups (study vs. control) and in terms of gender distribution. We noticed higher mean values for CTX2 $(763,74 \mathrm{pg} / \mathrm{mL}$ vs. $756,24 \mathrm{pg} / \mathrm{mL})$ and COMP $(36,83 \mathrm{ng} / \mathrm{mL}$ vs. 30,61 $\mathrm{ng} / \mathrm{mL}$ ) in the study group, even though the observed differences were not statistically significant. However, the above observation does not prejudge the high sensitivity of the above markers in relation to degenerative spine disease, because the values beyond the reference range were also observed in the control group. Therefore, the advancement of degenerative changes in the intervertebral disc cannot be determined by measuring the concentration of bone turnover markers in the blood serum. Degenerative disease of the lumbar spine is mainly local. For this reason, the determination of the levels of markers of bone and cartilage remodeling will probably be only justified in the synovial fluid, as described in the studies cited earlier. Examining the importance of bone turnover markers in terms of a specific disease, the patient's age and sex should be taken into account. Menopause appears to be a turning point in the assessment of the level of these proteins. Determination of bone turnover markers in a particular metabolic bone disease should be precisely specified. Research should be narrowed down to a specific group of people, with a narrow age range, equal gender distribution and a similar background survey. The biological characteristics of each marker should also be taken into account, in particular their diurnal variability, half-life and stability at room temperature. The type and timing of sample collection seem to be of greatest importance, as well as confounding factors such as food intake and comorbidities (liver diseases, kidney failure). Most of the studies conducted so far have focused on the importance of a specific marker in selected metabolic bone disease. In the diagnosis and monitoring of Paget's disease, measurement of the concentration of bone alkaline phosphatase (BALP) in the blood serum is used.[49] In osteoporosis, the markers currently measured include markers of bone formation - procollagen type $1 \mathrm{~N}$-terminal propeptide (P1NP), and markers of bone resorption, including cross-linked C-telopeptide of type 1 collagen (CTX1).[19] In neoplastic diseases whose course involves the skeletal system (prostate cancer, breast cancer), the level of tartrate-resistant acid tartrate-resistant acid phosphatase 5b (TRAP-5b) is determined.[50,51] There are many reports on the usefulness of NTX1, CTX1, CTX2, COMP in degenerative joint diseases. In each of the above-mentioned metabolic diseases of the bones, the level of calcium and vitamin $\mathrm{D}$ shall be additionally determined. Their deficiency is most often associated with the progression of the above diseases. Summarizing the above reports and the results of our research, it can certainly be stated that bone turnover markers are reliable indicators of bone and cartilage tissue remodeling. This applies not only to 
common metabolic bone diseases but also to degenerative spine disease. The presence of these proteins in the blood serum of every human is the result of numerous metabolic processes occurring at every stage of life. Beginning with intensive growth in adolescence and ending in old age. Their routine determination in the future may allow to indicate the severity of the degenerative spine disease, predict and assess the risk of pathological fractures and monitor the effectiveness of treatment. However, further research is required to verify their role in the pathogenesis of diseases with high bone turnover. This study aimed to determine the diagnostic usefulness of the previously discussed markers of bone turnover, cartilage damage and inflammation in degenerative disease of the lumbar spine. It appears that the diagnostic panel for degenerative disease of the lumbar spine should include: back pain questionnaire surveys, imaging tests (first overall or targeted X-ray of the spine and MRI subsequently) and laboratory tests (calcium, vitamin D and CRP protein levels). People with severe back pain due to the degenerative disease itself and its high degree of advancement in imaging studies are more and more frequently treated surgically than conservatively. This significantly improves the quality of life, resulting not only from the improvement in mobility or range of movements of the spine but primarily from pain relief. Back pain is the most important clinical indicator of degenerative spine disease, requires strict control and should not be underestimated, as it can be harmful in terms of medical, emotional, social and psychosocial aspects. The World Health Organization lists osteoarthritis among the top 10 health problems. Approach to the degenerative spine disease solely in the diagnostic aspect is insufficient. Particular attention must be focused on preventive measures, primarily oriented towards the population of people from risk groups. In the light of the current recommendations, prophylaxis should consist of educating patients and their families, regularly performing exercises to strengthen the core muscles and maintaining proper body weight. Weight reduction remains the standard management in degenerative disease of the lumbar spine, regardless of surgery and pharmacotherapy. People with back pain should be encouraged to stop smoking and drinking alcohol and advised that there is no benefit in taking dietary supplements. The change in the lifestyle alone in most patients with degenerative disease of the lumbar spine leads to a noticeable improvement in well-being and pain relief.[52,53,54]

\section{Conclusions}

1. People with diagnosed degenerative disease of the lumbar spine have higher levels of vitamin $\mathrm{D}$ and calcium in the blood serum than healthy people.

2. Vitamin D deficiency may be one of the risk factors for the development of the degenerative disease of the lumbar spine.

3. People with diagnosed degenerative disease of the lumbar spine show a higher concentration of CRP protein than healthy people.

4. Healthy people and people suffering from degenerative disease of the lumbar spine have comparable concentrations of P1NP, CTX2, COMP, and PTH in the blood serum. The determination of the above markers in the blood serum does not confer an advantage in the diagnosis and monitoring of degenerative spine disease.

\section{Literature}

1. Wasiak R.; Kim J.Y.; Pransky G. Work disability and costs caused by recurrence of low back pain: longer and more costly than in first episodes: Spine 2006, 31(2): 219-225.

2. Sarah E.E. Mills; Karen P. Nicolson; Blair H. Smith. Chronic pain: a review of its epidemiology and associated factors in population-based studies.Br J Anaesth 2019, 123(2): 273-283.

3. Wong A.Y.; Karppinen J.; Samartzis D. Low back pain in older adults: risk factors, management options and future directions. Scoliosis Spinal Disord. 2017, 12: 14.

4. Jing Yu; Peter C.; Sally Roberts; Jill P.G. Urban. Elastic fiber organization in the intervertebral discs of the bovine tail. J Anat. 2002, 201(6): 465-475.

5. Choi Y.S. Pathophysiology of degenerative disc disease. Asian Spine J. 2009, 3(1): 39-44.

6. Mundy G.R. Metastasis to the bone: causes, consequences and therapeutic opportunities. Nat Rev Cancer. 2002, 2(8): 584-593.

7. Bilsky M.H. New therapeutics in spine metastases. Expert Rev Neurother. 2005, 5(6): 831-840.

8. Roughley P.J. Biology of intervertebral disc aging and degeneration: involvement of the extracellular matrix. Spine 2004, 29(23): 2691-2699. 
9. Chris Daly; Peter Ghosh; Graham Jenkin; David Oehme; Tony Goldschlager. A Review of Animal Models of Intervertebral Disc Degeneration: Pathophysiology, Regeneration, and Translation to the Clinic. Biomed Res Int. 2016, 2016: 5952165.

10. Jill P.G. Urban, Sally Roberts. Degeneration of the intervertebral disc. Arthritis Research \& Therapy 2003, 5: 120-130.

11. Fujiwara A.; Tamai K.; An H.S.; Kurihashi T.; Lim T.H.; Yoshida H.; Saotome K. The relationship between disc degeneration, facet joint osteoarthritis, and stability of the degenerative lumbar spine. J Spinal Disord. 2000, 13(5): 444-450.

12. Vernon-Roberts B.; Moore R.J.; Fraser R.D. The natural history of age-related disc degeneration: the pathology and sequelae of tears. Spine 2007, 32(25): 2797-2804.

13. Haefeli M.; Kalberer F.; Saegesser D.; Nerlich A.G.; Boos N.; Paesold G. The course of macroscopic degeneration in the human lumbar intervertebral disc. Spine 2006, 31(14): 1522-1531.

14. Inoue N.; Espinoza Orías A.A. Biomechanics of intervertebral disk degeneration. Orthop Clin North Am. 2011, 42(4): 487-499.

15. Lafian A.M.; Torralba K.D. Lumbar Spinal Stenosis in Older Adults in Older Adults. Rheum Dis Clin North Am. 2018, 44(3): 501-512.

16. Greenblatt M.B.; Tsai J.N.; Wein M.N. Bone Turnover Markers in the Diagnosis and Monitoring of Metabolic Bone Disease. Clin Chem. 2017, 63(2): 464-474.

17. Vasikaran S.D.; Chubb S.P.; Ebeling P.R.; Jenkins N.; Jones G.R.; Kotowicz M.A.; Morris H.A.; Schneider H.G.; Seibel M.J.; Ward G. Harmonised Australian Reference Intervals for Serum PINP and CTX in Adults. Clin Biochem Rev. 2014, 35(4): 237-242.

18. Koivula M.K.; Risteli L.; Risteli J. Measurement of aminoterminal propeptide of type I procollagen (PINP) in serum. Clin Biochem Rev. 2012, 45(12): 920-927.

19. Mattias Lorentzon; Jaime Branco; Maria Luisa Brandi; Olivier Bruyère; Roland Chapurlat; Cyrus Cooper; Bernard Cortet; Adolfo Diez-Perez; Serge Ferrari; Andrea Gasparik; Markus Herrmann; Niklas Rye Jorgensen; John Kanis; Jean-Marc Kaufman; Andrea Laslop; Médéa Locquet; Radmila Matijevic; Eugene McCloskey; Salvatore Minisola; Richard Pikner; Jean-Yves Reginster; René Rizzoli; Pawel Szulc; Mila Vlaskovska; Etienne Cavalier. Algorithm for the Use of Biochemical Markers of Bone Turnover in the Diagnosis, Assessment and Follow-Up of Treatment for Osteoporosis. Adv Ther. 2019, 36(10): 2811-2824.

20. Andrzej Kutner; Geoffrey Brown. Vitamins D: Relationship between Structure and Biological Activity. Int J Mol Sci. 2018, 19(7): 2119.

21. Wiciński M.; Adamkiewicz D.; Adamkiewicz M.; Śniegocki M.; Podhorecka M.; Szychta P.; Malinowski B. Impact of Vitamin D on Physical Efficiency and Exercise Performance - A Review. Nutrients 2019, 11(11): 2826.

22. Hardcastle S.A.; Dieppe P.; Gregson C.L.; Davey Smith G.; Tobias J.H. Osteoarthritis and bone mineral density: are strong bones bad for joints? Bonekey Rep. 2015, 4: 624.

23. Clara Yongjoo Park. Vitamin D in the Prevention and Treatment of Osteoarthritis: From Clinical Interventions to Cellular Evidence. Nutrients 2019, 11(2): 243.

24. Goltzman D. Physiology of Parathyroid Hormone. Endocrinol Metab Clin North Am. 2018, 47(4): 743-758.

25. Lombardi G.; Di Somma C.; Rubino M.; Faggiano A.; Vuolo L.; Guerra E.; Contaldi P.; Savastano S.; Colao A. The roles of parathyroid hormone in bone remodeling: prospects for novel therapeutics. J Endocrinol Invest. 2011, 34(7 Suppl): 18-22.

26. Zanocco K.A.; Yeh M.W. Primary Hyperparathyroidism: Effects on Bone Health. Endocrinol Metab Clin North Am. 2017, 46(1): 87-104.

27. Orwoll E.S.; Scheele W.H.; Paul S.; Adami S.; Syversen U.; Diez-Perez A.; Kaufman J.M.; Clancy A.D.; Gaich G.A. The effect of teriparatide [human parathyroid hormone (1-34)] therapy on bone density in men with osteoporosis. J Bone Miner Res. 2003, 18(1): 9-17.

28. Krege J.H.; Lane N.E.; Harris J.M.; Miller P.D. PINP as a biological response marker during teriparatide treatment for osteoporosis. Osteoporos Int. 2014, 25(9): 2159-2171.

29. Espanha M.M. Articular cartilage: structure and histochemical composition. Tissue Eng Part B Rev. 2010, 16(6): 617-627.

30. Responte D.J.; Natoli R.M.; Athanasiou K.A. Collagens of articular cartilage: structure, function, and importance in tissue engineering. Crit Rev Biomed Eng. 2007, 35(5): 363-411. 
31. Posey K.L.; Coustry F.; Hecht J.T. Cartilage oligomeric matrix protein: COMPopathies and beyond: Matrix Biol. 2018, 71-72: 161-173.

32. Mingjian Huang; Jingyu Zhao; Yan Huang; Liming Dai; Xiaoling Zhang. Meta-analysis of urinary C-terminal telopeptide of type II collagen as a biomarker in osteoarthritis diagnosis. J Orthop Translat. 2018, 13: 50-57.

33. Garnero P.; Piperno M.; Gineyts E.; Christgau S.; Delmas P.D.; Vignon E. Cross sectional evaluation of biochemical markers of bone, cartilage, and synovial tissue metabolism in patients with knee osteoarthritis: relations with disease activity and joint damage. Ann Rheum Dis. 2001, 60(6): 619-626.

34. Pengkun Wang; Jinlong Song; Dongyang Qian. CTX-II and YKL-40 in early diagnosis and treatment evaluation of osteoarthritis. Exp Ther Med. 2019, 17(1): 423-431.

35. Young-Min S.A.; Cawston T.E.; Griffiths I.D. Markers of joint destructions: principles problems and potential. Ann Rheum Dis. 2001, 60(6): 545-548.

36. Garnero P.; Piperno M.; Gineyts E.; Christgau S.; Delmas P.D.; Vignon E. Cross sectional evaluation of biochemical markers of bone, cartilage, and synovial tissue metabolism in patients with knee osteoarthritis: relations with disease activity and joint damage. Ann Rheum Dis. 2001, 60(6): 619-626.

37. Dragomir A.D.; Kraus V.B.; Renner J.B.; Luta G.; Clark A.; Vilim V.; Hochberg M.C.; Helmick C.G.; Jordan J.M. Serum cartilage oligomeric matrix protein and clinical signs and symptoms of potential pre-radiographic hip and knee pathology. Osteoarthritis Cartilage. 2002, 10(9): 687-691.

38. Vilím V.; Olejárová M.; Machácek S.; Gatterová J.; Kraus V.B.; Pavelka K. Serum levels of cartilage oligomeric matrix protein (COMP) correlate with radiographic progression of knee osteoarthritis. Osteoarthritis Cartilage. 2002, 10(9): 707-713.

39. Garnero P.; Conrozier T.; Christgau S. Urinary type collagen C-propeptide levels in patients with rapidly destructive hip osteoarthritis. Ann. Rheum Dis. 2003, 62(10): 939-943.

40. Garnero P.; Ayral X.; Rousseau J.C.; Christgau S.; Sandell L.J.; Dougados M.; Delmas P.D. Uncoupling of type II collagen synthesis and degradation predicts progression of joint damage in patients with knee osteoarthritis. Arthritis Rheum. 2002, 46(10): 2613-2624.

41. Reijman M.; Hazes J.M.; Bierma-Zeinstra S.M.; Koes B.W.; Christgau S.; Christiansen C.; Uitterlinden A.G.; Pols H.A. A new marker of osteoarthritis: cross-selectional and longitudinal approach. Arthritis Rheum. 2004, 50(8): 2471-2478.

42. Garnero P.; Landewé R.; Boers M.; Verhoeven A.; Van Der Linden S.; Christgau S.; Van Der Heijde D.; Boonen A.; Geusens P. Association of baseline levels of markers of bone and cartilage degradation with long-term progression of joint damage in patients with early rheumatoid arthritis: the COBRA study. Arthritis Rheum. 2002, 46(11): 2847-2856.

43. Jung M.; Christgau S.; Lukoschek M.; Henriksen D.; Richter W. Increased urinary concentration of collagen type II C-telopeptide fragments in patients with osteoarthritis. Pathobiology 2004, 71(2): 70-76.

44. Lohmander L.S.; Atley L.M.; Pietka T.A.; Eyre D.R. The release of cross-linked peptides from type II collagen into human synovial fluid is increased soon after joint injury and in osteoarthritis. Arthritis Rheum. 2003, 48(11): 3130-3139.

45. Holick M.F.; Chen T.C. Vitamin D deficiency: a worldwide problem with health consequences. Am J Clin Nutr. 2008, 87(4): 1080-1086.

46. Pereira-Santos M.; Costa P.R.; Assis A.M.; Santos C.A.; Santos D.B. Obesity and vitamin D deficiency: a systematic review and meta-analysis. Obes Rev. 2015, 16(4): 341-349.

47. Glover T.L.; Goodin B.R.; King C.D.; Sibille K.T.; Herbert M.S.; Sotolongo A.S.; Cruz-Almeida Y.; Bartley E.J.; Bulls H.W.; Horgas A.L.; Redden D.T.; Riley J.L. $3^{\text {rd }}$ S Staud R.; Fessler B.J.; Bradley L.A.; Fillingim R.B. A Cross-sectional Examination of Vitamin D, Obesity, and Measures of Pain and Function in Middle-aged and Older Adults With Knee Osteoarthritis. Clin J Pain. 2015, 31(12): 1060-1067.

48. Zhang F.F.; Driban J.B.; Lo G.H.; Price L.L.; Booth S.; Eaton C.B.; Lu B.; Nevitt M.; Jackson B.; Garganta C.; Hochberg M.; Kwoh K.; McAlindon T.E. Vitamin D deficiency is associated with progression of knee osteoarthritis. J Nutr. 2014, 144(12): 2002-2008.

49. Kravets I. Paget's Disease of Bone: Diagnosis and Treatment. Am J Med. 2018, 131(11): 1298-1303.

50. Lyubimova N.V.; Pashkov M.V.; Tyulyandin S.A.; Gol'dberg V.E.; Kushlinskii N.E. Tartrate-resistant acid phosphatase as a marker of bone metastases in patients with breast cancer and prostate cancer. Bull Exp Biol Med. 2004, 138(1): 77-79. 
51. Reithmeier A.; Norgard M.; Ek-Rylander B.; Näreoja T.; Andersson G. Cathepsin K regulates localization and secretion of Tartrate-Resistant Acid Phosphatase (TRAP) in TRAP - over expressing MDA-MB-231 breast cancer cells. BMC Mol Cell Biol. 2020, 21(1): 15.

52. J. Towheed T.; Welch V.; Wells G.; Tugwell P. American College of Rheumatology; Arthritis Care Res (Hoboken). 2012, 64(4): 465-474.

53. Holger Joswig; Martin N. Stienen; Nicolas R. Smoll; Marco V. Corniola; Ivan Chau; Karl Schaller; Gerhard Hildebrandt; Oliver P. Gautschi. Effects of Smoking on Subjective and Objective Measures of Pain Intensity, Functional Impairment, and Health-Related Quality of Life in Lumbar Degenerative Disk Disease. World Neurosurg. 2017, 99:6-13.

54. Vincent, H.K.; Heywood K.; Connelly J.; Hurley R.W. Obesity and Weight Loss in the treatment and prevention of osteoarthritis. PM R. 2012, 4(5 Suppl): 59-67. 